\title{
Early diagnosis and effective treatment of endogenous and exogenous cesarean scar pregnancies: A retrospective case series.
}

\author{
Huanling Liu ${ }^{*}$, Yingxiong $\mathrm{Li}^{2}$, Dexiang Liu ${ }^{3}$, Shuxin Wang ${ }^{3}$, Ye Chen², Ruixia Wu ${ }^{2}$, Yujing Chen ${ }^{1}$, \\ Hanwei Chen ${ }^{3}$ \\ ${ }^{1}$ Department of Ultrasound, Guangzhou Panyu Central Hospital, PR China \\ ${ }^{2}$ Department of Gynecology and Obstetrics, Guangzhou Panyu Central Hospital, PR China \\ ${ }^{3}$ Department of Radiology, Guangzhou Panyu Central Hospital, PR China
}

\begin{abstract}
Objective: To evaluate the diagnostic accuracy of and the efficacy, safety, and cost effectiveness of treatment for endogenous and exogenous cesarean scar pregnancies (CSP). CSPs result from the amniotic sac implanting into the CS; endogenous CSPs grow toward the uterine cavity, while exogenous CSPs infiltrate the uterine myometrium.

Methods: Sixty nine (69) endogenous and exogenous CSP patients were retrospectively reviewed. The former group included two subgroups: dilation and curettage (D\&C) alone, and combined D\&C with uterine artery embolization (UAE). Treatment type, complications, and treatment costs were recorded. Means and standard deviations were calculated for all variables for each group and sub-group. Independent t-tests were conducted to compare all variables between the groups and subgroups.

Results: Sixty nine (69) patients were reviewed: 47 endogenous and 22 exogenous CSPs. 41 endogenous CSPs underwent ultrasound-guided D\&C. Two had complications and underwent surgical resections. The 6 remaining endogenous CSPs successfully underwent UAE and hysteroscopy-guided D\&C. Patients receiving the combined treatment did have significantly higher expenses $(\mathbf{P}<\mathbf{0 . 0 5})$. All 22 exogenous CSPs successfully underwent surgical resections. The exogenous CSPs had significantly higher hospitalization expenses than the endogenous CSPs $(\mathbf{P}<0.05)$.

Conclusion: It is safe and cost effective to use ultrasound-guided D\&C for endogenous CSPs and surgical resections for exogenous CSPs.
\end{abstract}

Keywords: Ultrasonography, MRI, Dilation and curettage, Resection, Uterine artery embolization. Accepted on November 16, 2017

\section{Introduction}

A caesarean scar pregnancy (CSP) occurs when the embryo implants into the myometrium within a scar from a previous cesarean delivery. CSP is life-threatening because it is associated with a high risk of severe bleeding and uterine rupture [1]. Early diagnosis and treatment can prevent or decrease the severity of these complications. Due to the increasing use of cesarean sections, the incidence of CSP is on the rise. A recent case series estimated that $0.15 \%$ of women who have previously had cesarean sections will have a CSP [2]. The incidence of CSP is especially an issue in China where the caesarean section rate rose dramatically from $3.4 \%$ of all deliveries in 1988 to $39.3 \%$ in 2008 due to China's previous policy of allowing urban families to have only one child. Under this policy urban women in China opted for cesarean sections because they perceived it to be the safer delivery option [3]. However, because China has changed this policy in recent years, women who previously had cesarean sections are becoming pregnant again, leading to a rise in CSP. Wu et al. reported that at their institutions, CSP has increased from 7.8 cases/100,000 pregnant women in 2000 to 36.2 cases/100,000 in 2010 [4]. Therefore, it is imperative to promote early diagnosis of CSP and to define appropriate treatment.

Vial et al. classified CSP into two types: endogenous and exogenous [5]. Endogenous CSP is caused by implantation of the amniotic sac into the previous cesarean scar (CS) with progression of the pregnancy toward the cervical isthmic space and the uterine cavity. Some authors have claimed that ultrasound-guided suction curettage can successfully treat endogenous CSP without the need for additional therapy [6-9]. Exogenous CSP is caused by deep implantation into a CS defect with growth infiltrating into the uterine myometrium and bulging into the uterine serosa and bladder. Currently there is no consensus regarding treatment for either type of CSP. Uterine curettage, methotrexate (MTX), surgical management, expectant management, and combined treatments have all been 
described in the literature as viable treatment options [10]. This lack of consensus has led to both overtreatment and insufficient treatment of early CSPs. Established diagnostic and treatment methods are needed. The goal of this study was to evaluate diagnostic accuracy regarding CSP and the efficacy, safety, and cost effectiveness of various treatments for CSP.

\section{Methods}

This retrospective study was approved by our institution's ethics committee; the need for informed consent was waived as this is a retrospective study. We used electronic medical records to calculate the number of newborns born at our institution between January 2010 and December 2014, the number and rate of cesarean sections from January 2010December 2014 and from 1980-1984, and the rate of CSPs during both aforementioned time periods. An electronic medical record search using the diagnosis "CSP" was conducted to identify potential patients. We identified a total of 87 CSP patients admitted to our institution between January 2010 and December 2014. Medical records and imaging results were then reviewed to find patients fitting the following inclusion criteria: (1) history of a cesarean delivery; (2) amenorrhea; (3) positive urine pregnancy test; (4) color Doppler trans-vaginal ultrasound (US) indicating an empty uterine cavity with a gestational sac located anteriorly to the uterus at the level of the lower uterine cesarean section scar that demonstrated rich perfusion surrounding the gestational sac or, for patients whose ultrasound findings did not clearly show the boundary between the gestational sac and the bladder, a magnetic resonance (MR) image detecting a thin or defective myometrium of the lower anterior uterine wall. Patients who received any treatment for CSP before hospital admission were excluded. Patients with coagulation disorders as well as trophoblastic diseases were also excluded.

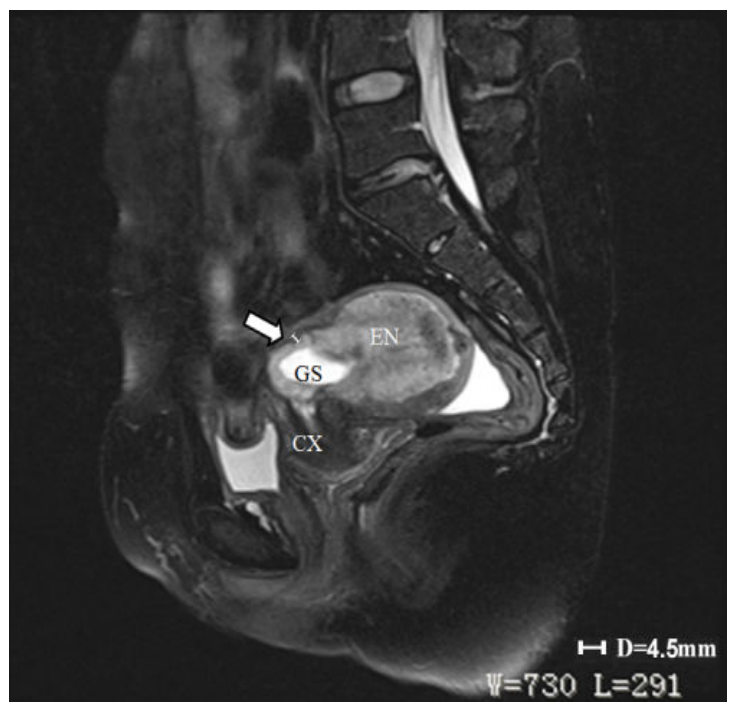

Figure 1. Sagittal T2-weighted MRI of the pelvis demonstrating a gestational sac located in a previous cesarean section scar with a thin myometrium (thickness=0.4 cm, long white arrow); the sac is growing toward the myometrium and the uterine cavity simultaneously indicating an endogenous CSP.

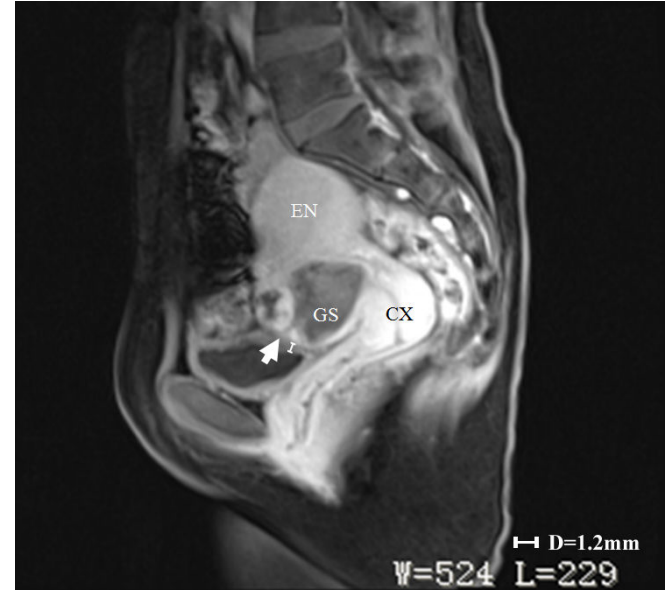

Figure 2. Sagittal T1-weighted enhanced MRI demonstrates a gestational sac of an exogenous CSP located deep in the scar and growing toward the myometrium and uterine cavity simultaneously; the thickness between the sac and bladder is $0.1 \mathrm{~cm}$ (short white arrow). (EN, endometrium; CX, cervix; GS, Gestational Sac.).

In addition to the criteria from Vial et al. mentioned in the introduction, endogenous and exogenous CSPs were distinguished from each other by evaluating the thickness of the lower anterior uterine wall on US and/or magnetic resonance imaging (MRI) [5]. A thickness greater than $0.3 \mathrm{~cm}$ was classified as endogenous (Figure 1); a thickness of $0.3 \mathrm{~cm}$ or less was classified as exogenous (Figure 2). Each patient's treatment type and course were reviewed, as well as the histological results of their CSP. The following data on each included patient were collected: patient age, gestational age at diagnosis, number of previous cesarean sections, preoperative vaginal bleeding, preoperative serum $\beta$-hCG level (mIU/ml), thickness of anterior lower uterine wall on US or MRI, gestational sac diameter on US or MRI, procedure time, perioperative blood loss, perioperative complications, length of postoperative hospital stay, treatment costs, and outcome assessment. Treatments were regarded as successful if there were no complications, no requirement to re-treat, and $\beta$-hCG returned to normal levels post-treatment within 21 days.

\section{Statistical analyses}

The means and standard deviations (SD) for all variables were calculated for each group and each sub-group. All data are expressed as mean $\pm \mathrm{SD}$. Independent sample t-test were used for intergroup comparisons. $\mathrm{P}<0.05$ was considered statistically significant. Statistical analyses were performed with version 18.0 SPSS statistical software (SPSS Inc., Chicago, IL, USA).

\section{Results}

From January 2010 to December 2014, 25,701 newborns were delivered at our institution; 12,346 (48\%) were via cesarean section in contrast to $4.7 \%$ between 1980 and 1984 . Concurrently, our institutional CSP rate increased from 0.17 cases/1,000 pregnant women in 1980-1984 to 1.8 cases/1,000 in 2010-2014. In fact, 87 patients between January 2010 and December 2014 were diagnosed with CSP. In the present study, 
we excluded 16 patients for receiving CSP treatment before their admission. Two patients were also excluded for having a coagulation disorder. Therefore, 69 subjects fulfilled the inclusion criteria. 47 subjects were diagnosed with endogenous CSPs while 22 were diagnosed with exogenous CSPs. 56 patients received MRI exams because their ultrasound findings were inconclusive. Demographics, clinical characteristics, and patient outcomes of both groups are listed in Table 1. There were no significant differences between the endogenous and exogenous CSP groups regarding patients' age, gestational age, gestational sac diameter, number of previous cesarean sections, preoperative serum $\beta$-hCG level $(\mathrm{mIU} / \mathrm{mL})$, perioperative blood loss, length of hospital stay, and success rates. The procedure times and hospitalization expenses were significantly lower in the endogenous group than in the exogenous group $(5899.45 \pm 1456.87$ (RMB) versus 15822.80 $\pm 4541.85(\mathrm{RMB})(\mathrm{P}<0.05)$, respectively).

Table 1. Comparison of demographics, clinical characteristics, and outcomes between the endogenous CSP and exogenous CSP groups.

\begin{tabular}{|c|c|c|c|}
\hline Type & $\begin{array}{l}\text { Endogenous CSP } \\
(n=47)\end{array}$ & $\begin{array}{l}\text { Exogenous CSP } \\
(n=22)\end{array}$ & $P$ value \\
\hline Age (years) & $32.82 \pm 3.01$ & $33.24 \pm 4.25$ & 0.43 \\
\hline $\begin{array}{l}\text { \# of previous cesarean } \\
\text { sections/patient }(n)\end{array}$ & $1.31 \pm 0.62$ & $1.89 \pm 0.86$ & 0.36 \\
\hline Gestational age (days) & $52.13 \pm 5.56$ & $56.82 \pm 6.91$ & 0.30 \\
\hline $\begin{array}{l}\text { Gestational sac diameter } \\
(\mathrm{cm})\end{array}$ & $3.56 \pm 2.89$ & $4.61 \pm 1.92$ & 0.23 \\
\hline $\begin{array}{l}\text { Preoperative } \quad \text { vaginal } \\
\text { bleeding }(\mathrm{ml})\end{array}$ & $88.72 \pm 68.23$ & $96.65 \pm 56.45$ & 0.32 \\
\hline $\begin{array}{l}\text { Perioperative serum } \beta \text { - } \\
\text { hCG level }(\mathrm{mlU} / \mathrm{mL})\end{array}$ & $87.00-25468.50$ & $107.50-53349.00$ & 0.12 \\
\hline $\begin{array}{l}\text { Thickness of lower anterior } \\
\text { uterine wall }(\mathrm{cm})\end{array}$ & $2.04 \pm 1.43$ & $0.28 \pm 0.12$ & $<0.05$ \\
\hline Procedure time (min) & $46.89 \pm 22.17$ & $70.98 \pm 31.57$ & $<0.05$ \\
\hline $\begin{array}{l}\text { Perioperative blood loss } \\
(\mathrm{ml})\end{array}$ & $108.54 \pm 79.76$ & $70.78 \pm 56.41$ & 0.32 \\
\hline Successful cases $(n, \%)$ & $45(95.7 \%)$ & $22(100 \%)$ & 0.33 \\
\hline $\begin{array}{l}\text { Length of hospital stay } \\
\text { (days) }\end{array}$ & $3.80 \pm 2.85$ & $5.05 \pm 2.05$ & 0.13 \\
\hline $\begin{array}{l}\text { Hospitalization expenses } \\
\text { (RMB) }\end{array}$ & $5899.45 \pm 1456.87$ & $\begin{array}{l}15822.80 \\
4541.85\end{array}$ & $<0.05$ \\
\hline
\end{tabular}

Table 2. Comparison of the demographics, clinical characteristics and outcomes between $D \& C$ and $U A E+D \& C$ in endogenous CSPs.

\begin{tabular}{llll}
\hline Item & D\&C $(\mathbf{n}=\mathbf{4 1})$ & UAE+D\&C $(\mathbf{n = 6})$ & P value \\
\hline Age (years) & $32.23 \pm 3.13$ & $33.33 \pm 2.96$ & 0.35 \\
\hline $\begin{array}{l}\text { \# of previous cesarean } \\
\text { sections/patient }(\mathrm{n})\end{array}$ & $1.26 \pm 0.34$ & $1.36 \pm 0.45$ & 0.38 \\
\hline $\begin{array}{l}\text { Gestational age (days) } \\
\text { Gestational sac diameter }\end{array}$ & $2.44 \pm 1.83$ & $4.63 \pm 2.94$ & 0.32 \\
$(\mathrm{~cm})$
\end{tabular}

\begin{tabular}{|c|c|c|c|}
\hline $\begin{array}{l}\text { Preoperative } \\
\text { bleeding }(\mathrm{ml})\end{array}$ & $25.66 \pm 17.25$ & $256.85 \pm 189.85$ & $<0.05$ \\
\hline $\begin{array}{l}\text { Perioperative Serum } \beta \text { - } \\
\text { hCG level }(\mathrm{mlU} / \mathrm{ml})\end{array}$ & $87.00-14876.05$ & $898.05-25468.05$ & $<0.05$ \\
\hline $\begin{array}{l}\text { Thickness of lower anterior } \\
\text { uterine wall }(\mathrm{cm})\end{array}$ & $2.22 \pm 1.63$ & $0.84 \pm 0.53$ & $<0.05$ \\
\hline Procedure time $(\mathrm{min})$ & $42.31 \pm 27.82$ & $70.95 \pm 31.58$ & $<0.05$ \\
\hline $\begin{array}{l}\text { Perioperative blood loss } \\
(\mathrm{ml})\end{array}$ & $108.62 \pm 69.76$ & $96.67 \pm 59.48$ & 0.42 \\
\hline $\begin{array}{l}\text { Successful cases, rate ( } \mathrm{n} \text {, } \\
\%)\end{array}$ & $39(95.1 \%)$ & $6(100 \%)$ & 0.37 \\
\hline $\begin{array}{l}\text { Length of hospital stay } \\
\text { (days) }\end{array}$ & $3.2 \pm 1.8$ & $5.1 \pm 2.6$ & 0.23 \\
\hline $\begin{array}{l}\text { Hospitalization expenses } \\
\text { (RMB) }\end{array}$ & $3236.45 \pm 1211.55$ & $\begin{array}{l}12822.65 \\
3976.86\end{array}$ & $\pm<0.05$ \\
\hline
\end{tabular}

Of the 47 endogenous CSP patients, 41 underwent US-guided dilatation and curettage (D\&C) alone; the other 6 patients underwent uterine artery embolization (UAE) followed by hysteroscopic-guided D\&C. Of these 41 patients, 39 underwent successful procedures; 2 patients required a procedural conversion to transabdominal lesion resection and blood transfusions due to heavy vaginal bleeding during D\&C. The surgeons found that the anterior myometrium at the location of previous cesarean incision was defective and had replaced trophoblastic tissue, indicating that these patients had been misdiagnosed and actually had exogenous CSPs. These two patients' total perioperative blood losses were $1750 \mathrm{~mL}$ and $2200 \mathrm{~mL}$, respectively. They both required blood transfusions, but neither of them had further complications. The $6 \mathrm{UAE}$ with D\&C group patients had exhibited heavy vaginal bleeding $(>500 \mathrm{~mL}$ ) before treatment, a gestational diameter $>4 \mathrm{~cm}$, or a $\beta$-hCG level greater than $15,000 \mathrm{mIU} / \mathrm{mL}$. All procedures for these 6 patients were carried out successfully.

Demographics, clinical characteristics, and outcomes of the patients in the endogenous CSP subgroups are listed in Table 2. There were no statistically significant differences between the two subgroups regarding patient age, gestational age, gestational sac diameter, perioperative blood loss, length of hospital stay, and procedural success. The UAE and D\&C group had significantly more preoperative vaginal bleeding and higher preoperative serum $\beta$-hCG levels. The thickness of the lower anterior wall was significantly higher in the $\mathrm{D} \& \mathrm{C}$ alone group. The procedure length was significantly shorter in the $\mathrm{D} \& \mathrm{C}$ alone group, and the hospitalization expenses were significantly higher for the combined UAE and D\&C group $(\mathrm{P}<0.05)$. The 22 exogenous CSP patients underwent individualized surgical treatments: 14 had trans-vaginal resections, 3 had trans-abdominal resections, 1 had a hysteroscopic resection under ultrasound guidance, 1 had a laparoscopic resection, 1 had a UAE followed by trans-vaginal resection, and 3 had UAEs followed by trans-abdominal resections. Surgical management was successful in all cases. At the operation, the uterine scar appeared either thin or hard to detect. The different surgical methods and outcome of these cases are presented in Table 3. All patients' histological 
examinations confirmed the presence of a first trimester trophoblast.

Table 3. Outcomes of exogenous CSPs treated with different surgical methods.

\begin{tabular}{|c|c|c|c|c|c|c|}
\hline \#of patients & Treatment & Procedure time (min) & $\begin{array}{l}\text { Perioperative blood loss } \\
\text { (ml) }\end{array}$ & $\begin{array}{l}\text { Length of Hospital } \\
\text { Stay (days) }\end{array}$ & $\begin{array}{l}\text { Hospitalization } \\
\text { (RMB) }\end{array}$ & expenses \\
\hline 14 & Trans-vaginal resection & $52.54 \pm 21.63$ & $106.43 \pm 69.91$ & $6.14 \pm 2.33$ & $9273.5 \pm 2124.8$ & \\
\hline 3 & Trans-abdominal resection & $68.56 \pm 27.54$ & $101.43 \pm 45.63$ & $7.65 \pm 2.55$ & $6132.9 \pm 1432.8$ & \\
\hline 1 & Hysteroscopic resection & 56.0 & 65.0 & 4.0 & 17789.5 & \\
\hline 1 & Laparoscopic resection & 62.0 & 80.0 & 4.0 & 18263.0 & \\
\hline 3 & UAE+transabdominal resection & $89.6 \pm 39.4$ & $68.9 \pm 34.6$ & $7.45 \pm 2.34$ & $21347.8 \pm 2567.7$ & \\
\hline
\end{tabular}

\section{Discussion}

As stated above, our institutional CSP rate increased from 0.17 cases/1,000 pregnant women in 1980-1984 to 1.8 cases/1,000 in 2010-2014. This CSP trend extends across China and much of the word where the rate of cesarean sections has increased [3]. Therefore, it is critically important to establish appropriate diagnostic and treatment protocols for CSP. The development of US and MRI have made the early diagnosis of CSP possible and have allowed for a high success rate of conservative treatment [11]. However, we found that in some cases it is difficult to identify the type of CSP according to the classification criteria of Vial et al. [5]. For example, in Figure 2 , the gestational sac was located in the previous cesarean section scar and the sac was protruding into the myometrium and the uterine cavity simultaneously. Therefore, we added a criterion, supported by past studies, that if the thickness of the low anterior uterine wall is greater than $0.3 \mathrm{~cm}$, the CSP would be defined as endogenous $[6,7,9,12]$.

Traditional treatments of CSP include methotrexate, dilation and curettage, uterine artery embolization, and transvaginal or trans-abdominal lesion resection [13]. Treatment varies depending on the patient's preoperative status. In this study, we conducted a comprehensive retrospective review of preoperative factors that determine treatment including the CSP type, gestational sac diameter, serum $\beta$-hCG level, and vaginal bleeding. Based on these factors patients received D\&C alone or with UAE or surgery. Neither methotrexate treatments nor blind uterine curettage is performed at our institution. Systemic or locally injected methotrexate treatment requires long hospitalization and often leads to serious side effects [14]. Blind curettage as a primary treatment is often insufficient because it cannot precisely reach the gestational tissue and can cause life- threatening blood loss and uterine rupture [2]. Although some authors have reported suction curettage to be safe and effective in certain cases where the trophoblast had shallowly infiltrated the uterine myometrium and when the thickness between the sac and bladder was $>0.3-0.45 \mathrm{~cm}$, it is of our opinion that blind curettage should be discouraged $[6,7,9]$.

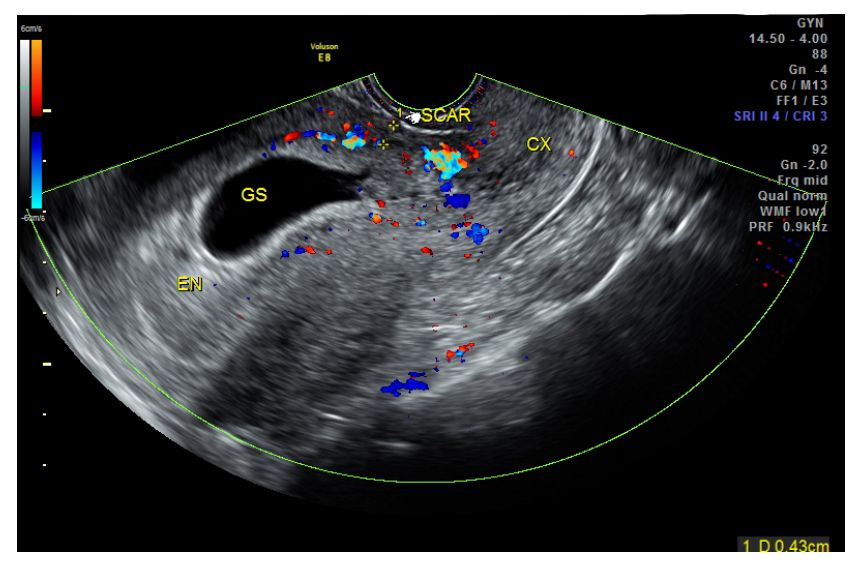

Figure 3. Trans-vaginal ultrasound image of a cesarean scar pregnancy at 9.2 weeks. The patient had severe bleeding of about $2000 \mathrm{~mL}$ during curettage. A gestational sac was implanted at the previous cesarean section scar. The thickness between the sac and bladder hard to detect (white arrow).

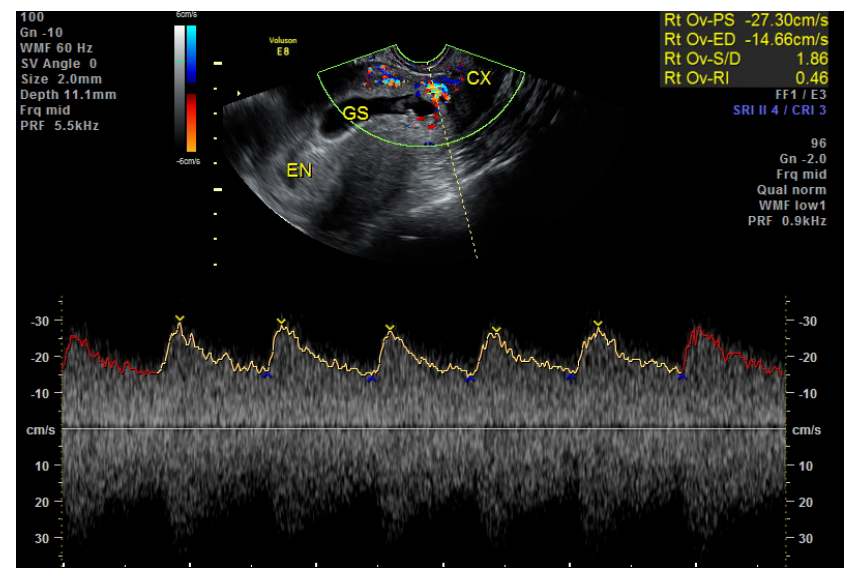

Figure 4. Color-flow Doppler of the same patient. Color Doppler imaging demonstrates rich perfusion surrounding the gestational sac.

In our study, 41 endogenous CSP patients were treated via D\&C under US guidance. 39 patients had successful evacuations. Two patients had heavy perioperative bleeding and had to then undergo trans-abdominal lesion resections and blood transfusions. They were then shown to have exogenous CSPs. The misdiagnoses were made because physicians were 
unable to identify the blurred bordering line of the anterior uterine myometrium and mistook the trophoblastic tissue for the myometrium on ultrasound (Figures 3 and 4). Therefore, accurate diagnosis is critical to ensure appropriate treatment. MRI is especially suitable to detect the myometrial thickness between the gestational sac and the bladder [15]. At our institution, MRI is performed on those patients who are suspected to have CSP but whose anatomy makes it difficult to identify the boundary between the sac and the bladder. Unfortunately the two patients who were misdiagnosed did not receive MRI exams; it is possible MRI exams would have shown that they had exogenous CSPs. Nevertheless, misdiagnosis is always a possibility; therefore we recommend preparations be made for a possible transfusion and emergency hysterectomy when performing D\&C. Furthermore, using trans-abdominal ultrasound guidance during the procedure can aid in detecting the location of the gestational sac and can help avoid uterine perforation and incomplete abortion.

There are circumstances under which D\&C alone is not an appropriate treatment for endogenous CSPs as massive hemorrhages during or after curettage have been reported [16]. When the gestational sac is $>4 \mathrm{~cm}$ in diameter, it is richly perfused with low vascular resistance or it has a lower anterior uterine wall thickness $<0.4 \mathrm{~cm}$; therefore, there is an increased risk of hemorrhage during $\mathrm{D} \& \mathrm{C}$ and an increased need to perform UAE to control the bleeding $[17,18]$. UAE and D\&C has been shown to be safe when UAE is the primary treatment followed by curettage; the success rate is 99.1-100\% [19]. Takeda et al. described five cases of CSP treated with UAE followed by curettage, showing that UAE is useful as an emergent intervention [20]. UAE for vessel occlusion followed by uterine curettage has been demonstrated to be more effective than D\&C alone with reduced blood loss, shorter $\beta$ hCG resolution time, and a shorter hospital stay [21]. Several studies suggest that this treatment would be effective in patients with $\beta$-hCG levels greater than $15,000 \mathrm{mIU} / \mathrm{mL}$ [22]. Hysteroscopy allows for the identification of the gestational sac and the visualization of the vessels at the implantation site [23]. Here we had 6 endogenous patients undergo UAE and D\&C under hysteroscopic guidance because they had excessive vaginal bleeding $(>500 \mathrm{~mL})$ before treatment, a gestational diameter $>4 \mathrm{~cm}$, or a $\beta$-hCG level greater than $15,000 \mathrm{mIU} / \mathrm{mL}$. All 6 patients were treated successfully, with a similar amount of blood loss and a similar length of hospital stay relative to those who received D\&C alone. However, the average costs were almost four times as much as $\mathrm{D} \& \mathrm{C}$ alone. We believe our findings contrast with those of Takeda et al. regarding length of hospital stay and blood loss due to our small sample size.

Regarding exogenous CSPs, Donnez et al. suggested that if the residual myometrium measures $<0.25 \mathrm{~cm}$, surgical repair should be performed [24]. In the 22 exogenous cases, the pregnancy sac was seen bulging through the low anterior uterine wall on MRI. Some lesions had evaginated significantly without a clear borderline of uterine myometrium. Because of the defects and severe impairments of the local myometrium in an exogenous CSP, the continuous presence of cesarean scar defects may cause rupture of the uterus. Surgical treatment offers the opportunity to remove the gestational mass and simultaneously repair the scar defect so that the risk of CSP recurrence is low. Thus, all 22 exogenous patients received surgical treatment at our institution. The variety of surgical treatments provided to different patients from 2010-2014 reflects the fact that surgical treatment for CSP developed during those four years. Laparoscopic surgery for a cesarean scar pregnancy has especially evolved at our institution in the last two years. It avoids open laparotomy, demonstrates a high success rate, fewer complications, and a shorter time for $\beta$-hCG levels to normalize. This procedure is especially suitable for the treatment of exogenous CSPs $[8,25]$. We treated 1 exogenous CSP with laparoscopic resection, which produced satisfactory results, including a shorter postoperative hospital stay and less trauma because an open laparotomy did not have to be performed [8].

Although trans-abdominal lesion resection can remove a CSP completely, repair the original scar, reduce the risk of recurrence, and reduce $\beta$-hCG levels rapidly, it has certain disadvantages, such as a large surgical incision, a risk for bladder injury, and a slower recovery [26]. However, the cost is lower than other surgical methods. Three patients chose this surgery because of the lower cost and recovered successfully. 14 patients were treated by transvaginal lesion resection. Typically, the cesarean section scar is located in the lower uterine segment, and lesions are usually deep in the pelvis, so it is hard to expose the focal area via the abdomen. Thus the trans-vaginal approach can be preferable. Compared with the transabdominal approach, the transvaginal resection procedure time is shorter, has less intraoperative blood loss, has a shorter postoperative hospital stay, and is not associated with bladder injury [26,27]. Combined UAE and trans-vaginal resection were performed in 3 patients who had heavy uterine bleeding $(>500 \mathrm{~mL})$. UAE is now accepted as a highly effective technique for controlling acute uterine bleeding during transvaginal lesion resection [28].

Hysteroscopic resection of CSP contents has been safely performed using US guidance [29]. Hysteroscopic lesion resection was done on 1 patient using ultrasound guidance. Although hysteroscopic resection of CSP is characterized by direct visualization and reliable resection of the lesions, the exogenous CSP gestational sac deeply implants adjacent to the myometrium, making it difficult during hysteroscopic surgery to completely remove the chorionic villi implanted in the myometrium. Clear hysteroscopic observation of the gestational sac, chorion, decidua, and the border between the gestational sac and the myometrium are possible after dilation of the cervical canal.

Based on our clinical experiences, we found it is critical to make an early and precise diagnose of CSP and to classify it accurately to prevent complications due to misdiagnosis and subsequently inappropriate treatment. All pregnant women with a previous history of cesarean scar should undergo transvaginal ultrasound to determine the implantation site of the gestational sac as early as possible; furthermore, MRI 
should be considered if the diagnosis or depth of invasion is in question. Recent efforts have also employed 3D US images as well as hysteroscopy to improve diagnostic abilities when it comes to CSP. Treatments should be individualized based on the specific characteristics of each CSP, its imaging features, and patient preferences. Our study's sample size for exogenous CSPS was small due to the study's retrospective nature and did not allow for comparisons between treatments. Further research is needed to compare the safety and cost-effectiveness of surgical methods and non-surgical methods to treat exogenous CSP. Finally, it is of utmost importance to strictly control indications for cesarean sections. CSPs can be prevented in the future by ensuring cesarean sections are only performed when medically necessary.

\section{Acknowledgment}

The authors would like to thank Department of Pathology and the Department of Statistics at Guangzhou Panyu Central Hospital for their assistance in conducting this study.

\section{References}

1. Rotas MA, Haberman S, Levgur M. Cesarean scar ectopic pregnancies: etiology, diagnosis, and management. Obstet Gynecol 2006; 107: 1373-1381.

2. Ash A, Smith A, Maxwell D. Caesarean scar pregnancy. BJOG 2007; 114: 253-263.

3. Feng XL, Xu L, Guo Y, Ronsmans C. Factors influencing rising caesarean section rates in China between 1988 and 2008. Bull World Health Organ 2012; 90: 30-39.

4. Wu X, Xue X, Wu X, Lin R. Combined laparoscopy and hysteroscopy vs. uterine curettage in the uterine artery embolization-based management of cesarean scar pregnancy: a cohort study. Int J Clin Exp Med 2014; 7: 2793-2803.

5. Vial Y, Petignat $P$, Hohlfeld P. Pregnancy in a cesarean scar. Ultrasound Obstet Gynecol 2000; 16: 592-593.

6. Arslan M, Pata O, Dilek TU, Aktas A, Aban M, Dilek S. Treatment of viable cesarean scar ectopic pregnancy with suction curettage. Int J Gynaecol Obstet 2005; 89: 163-166.

7. Bayoglu Tekin Y, Mete Ural U, Balik G, Ustuner I, Kir Sahin F, Guvendag Guven ES. Management of cesarean scar pregnancy with suction curettage: a report of four cases and review of the literature. Arch Gynecol Obstet 2014; 289: 1171-1175.

8. Wang G, Liu X, Bi F, Yin L, Sa R, Wang D. Evaluation of the efficacy of laparoscopic resection for the management of exogenous cesarean scar pregnancy. Fertil Steril 2014; 101: 1501-1507.

9. Weilin C, Li J. Successful treatment of endogenous cesarean scar pregnancies with transabdominal ultrasoundguided suction curettage alone. Eur J Obstet Gynecol Reprod Biol 2014; 183: 20-22.

10. McKenna DA, Poder L, Goldman M, Goldstein RB. Role of sonography in the recognition, assessment, and treatment of cesarean scar ectopic pregnancies. J Ultrasound Med 2008; 27: 779-783.

11. Iyibozkurt AC, Topuz S, Gungor F, Kalelioglu IH, Cigerli E, Akhan SE. Conservative treatment of an early ectopic pregnancy in a cesarean scar with systemic methotrexate-case report. Clin Exp Obstet Gynecol 2008; 35: 73-75.

12. Weimin W, Wenqing L. Effect of early pregnancy on a previous lower segment cesarean section scar. Int J Gynaecol Obstet 2002; 77: 201-207.

13. Fylstra DL. Ectopic pregnancy within a cesarean scar: a review. Obstet Gynecol Surv 2002; 57: 537-543.

14. Wang HY, Zhang J, Li YN, Wei W, Zhang DW, Lu YQ. Laparoscopic management or laparoscopy combined with transvaginal management of type II cesarean scar pregnancy. JSLS 2013; 17: 263-272.

15. Liu Q, Zhang R, Wang X, Liu K, Bai G, Huang Y. Diagnostic value of 3.0 T magnetic resonance imaging for early cesarean scar pregnancy. Zhonghua Yi Xue Za Zhi 2014; 94: 3589-3592.

16. Polat I, Alkis I, Sahbaz A, Sahin O, Ekiz A, Gulac B. Diagnosis and management of cesarean scar pregnancy. Clin Exp Obstet Gynecol 2012; 39: 365-368.

17. Ayoubi JM, Fanchin R, Meddoun M, Fernandez H, Pons JC. Conservative treatment of complicated cesarean scar pregnancy. Acta Obstet Gynecol Scand 2001; 80: 469-470.

18. Hois EL, Hibbeln JF, Alonzo MJ, Chen ME, Freimanis MG. Ectopic pregnancy in a cesarean section scar treated with intramuscular methotrexate and bilateral uterine artery embolization. J Clin Ultrasound 2008; 36: 123-127.

19. Wu X, Zhang X, Zhu J, Di W. Caesarean scar pregnancy: comparative efficacy and safety of treatment by uterine artery chemoembolization and systemic methotrexate injection. Eur J Obstet Gynecol Reprod Biol 2012; 161: 75-79.

20. Takeda A, Koyama K, Imoto S, Mori M, Nakano T, Nakamura H. Diagnostic multimodal imaging and therapeutic transcatheter arterial chemoembolization for conservative management of hemorrhagic cesarean scar pregnancy. Eur J Obstet Gynecol Reprod Biol 2010; 152: 152-156.

21. Li C, Li C, Feng D, Jia C, Liu B, Zhan X. Transcatheter arterial chemoembolization versus systemic methotrexate for the management of cesarean scar pregnancy. Int $\mathrm{J}$ Gynaecol Obstet 2011; 113: 178-182.

22. Maymon R, Halperin R, Mendlovic S, Schneider D, Herman A. Ectopic pregnancies in a Caesarean scar: review of the medical approach to an iatrogenic complication. Hum Reprod Update 2004; 10: 515-523.

23. Deans R, Abbott J. Hysteroscopic management of cesarean scar ectopic pregnancy. Fertil Steril 2010; 93: 1735-1740.

24. Donnez O, Jadoul P, Squifflet J, Donnez J. Laparoscopic repair of wide and deep uterine scar dehiscence after cesarean section. Fertil Steril 2008; 89: 974-980.

25. Wang YL, Su TH, Chen HS. Operative laparoscopy for unruptured ectopic pregnancy in a caesarean scar. BJOG 2006; 113: 1035-1038. 
Early diagnosis and effective treatment of endogenous and exogenous cesarean scar pregnancies: A retrospective case series

26. Wang DB, Chen YH, Zhang ZF, Chen P, Liu KR. Evaluation of the transvaginal resection of low-segment cesarean scar ectopic pregnancies. Fertil Steril 2014; 101: 602-606.

27. Lu HY, Zhang WH, Shan J, Tian QS, Zhang XQ, Wu LC. Study on 31 cases with cesarean scar pregnancy treated by transvaginal surgery. Zhonghua Fu Chan Ke Za Zhi 2011; 46: 917-922.

28. Liang F, He J. Methotrexate-based bilateral uterine arterial chemoembolization for treatment of cesarean scar pregnancy. Acta Obstet Gynecol Scand 2010; 89: 1592-1594.
29. Hoshino T, Miyamoto T, Yoshioka S. Resection of cesarean scar pregnancy at six weeks of gestation with laminaria cervical dilatation under sonographic and hysteroscopic guidance. Case Rep Obstet Gynecol 2015; 2015: 685-761.

\section{*Correspondence to}

Huanling Liu

Department of Ultrasound

Guangzhou Panyu Central Hospital

PR China 